ISSN 2073-4360

www.mdpi.com/journal/polymers

Article

\title{
Dependence of Optical and Microstructure Properties of Thiol-Capped Silver Nanoparticles Embedded in Polymeric Matrix
}

\author{
Angela Longo ${ }^{1, *}$, Gianfranco Carotenuto ${ }^{1}$, Mariano Palomba ${ }^{1}$ and Sergio De Nicola ${ }^{2}$ \\ 1 Institute for Composite and Biomedical Materials, National Research Council (IMCB-CNR), \\ P.le Tecchio 80, 80125 Naples, Italy; E-Mails: giancaro@unina.it (G.C.); \\ marianopalomba@fastwebnet.it (M.P.) \\ 2 National Institute of Optics, National Research Council (INO-CNR), Viale Campi Flegrei 34, \\ 80078 Pozzuoli, Italy; E-Mail: s.denicola@alice.it (S.D.N.) \\ * Author to whom correspondence should be addressed; E-Mail: anglongo@unina.it; \\ Tel.: +39-081-775-88-34; Fax: +39-081-775-88-50.
}

Received: 28 July 2011; in revised form: 28 September 2011 / Accepted: 12 October 2011 / Published: 13 October 2011

\begin{abstract}
Thiol-capped silver nanoparticles were prepared by in situ thermal decomposition of different silver(I)-thiolates precursors in a polymeric matrix. Depending on the structure of the organic coating, contact-free distribution of metal nanoparticles or nanoparticles aggregates were achieved. The structure and morphology of nanocomposites was analyzed by Transmission Electron Microscopy (TEM), and X-Ray Diffraction (XRD). Nanoparticles' interaction was investigated by differential scanning calorimetry (DSC), and UV-Visible spectroscopy. In particular, only silver nanoparticles coated by n-alkyl thiols aggregated, while a contact-free dispersion was obtained by cyclohexyl thiol-capped silver nanoparticles.
\end{abstract}

Keywords: metal-polymer nanocomposite; thiol-capped silver nanoparticles; interdigitation phenomena 


\section{Introduction}

Organic ligand-capped metal nanoparticles, generally described as metal clusters compounds, have attracted attention due to their fascinating properties and their application in different technological areas, including the fabrication of biological probes, single-electron transistors and novel catalysts [1-8]. The interest in these materials is related to their versatility. In fact, the organic ligand-capped produces a shell around the nanoparticle surface which stabilizes the nanoparticles (NPs) avoiding the coalescence phenomenon (i.e., metal core sintering) and allowing the NPs to be re-dispersed if desired.

Therefore, the synthesis of metal cluster compounds by spontaneous self-assembly of thiols (for example, normal alkyl-thiols, $\mathrm{HS}\left(\mathrm{CH}_{2}\right)_{n} \mathrm{X}$, where $n$ is the number of carbon atoms that form the chain length and $\mathrm{X}$ the end-group) on the metal nanoparticles surface has been extensively studied [2,9-12]. Thiols are well-known ligand molecules because they can form a robust self-assembled monolayer on the metal nanoparticle surfaces through a strong metal-sulfur covalent bond, and are also able to bind together via Van der Waals interaction [13]. For example, the energy gold-sulfur bond is $418 \mathrm{~kJ} \mathrm{~mol}^{-1}$ and the silver-sulfur bond is $217 \mathrm{~kJ} \mathrm{~mol}^{-1}$ [14].

With regard to these interactions, the interdigitation phenomenon is of great interest. This phenomenon is related to the interpenetration of the thiol chains present on the nanoparticle surface. The interdigitation leads to the formation of a close packed structure (see Figure 1) such as the organized superlattice, in which the interparticles distance $\boldsymbol{D}_{\boldsymbol{p} p}$, i.e., the distance between the metal cores, depends on the thiol chains length, $\boldsymbol{D}_{l}$ [2]. In fact, the interdigitation phenomenon depends on the chain's length and on the presence of the gauche defect and it counterbalances the lower density of chains packing at their ends [2].

Figure 1. Schematic representation of different interaction of thiol-capped silver nanoparticles.
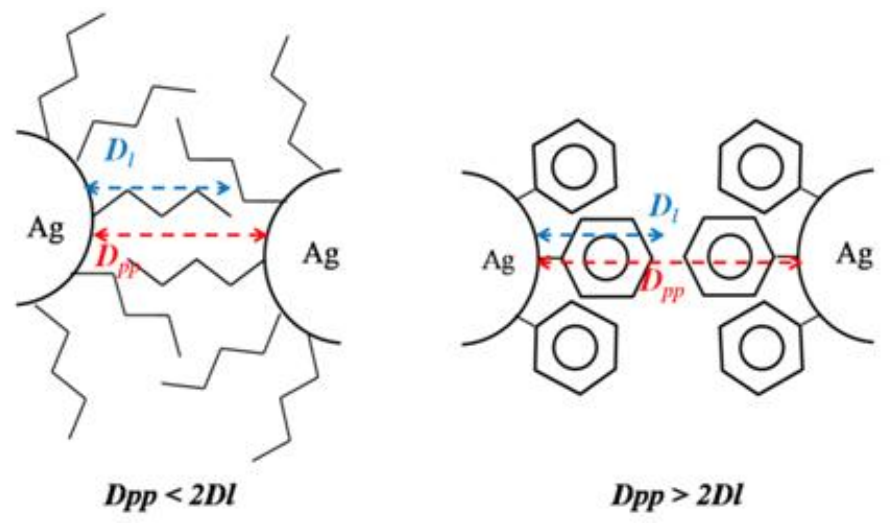

As a result of the interdigitation phenomenon, the interparticles distance, $\boldsymbol{D}_{\boldsymbol{p} p}$, halves by a factor of about 2 compared to the thiol chain length, $\boldsymbol{D}_{\boldsymbol{l}}$, and it decreases in a non-linear way, with increasing chain lengths. In fact, the interdigitation phenomenon is not observed for thiol chains with a length $\mathrm{n}<5$ or for steric encumbrance [2].

Usually, the metal nanoparticle surfaces are modified by passivation in order to improve their compatibility and affinity with another phase such as a polymeric matrix. For example, metal nanoparticles can prefer a polar or non-polar medium when appropriate organic groups are attached to 
the surface [15-17]. This aspect is very important in metal-polymer nanocomposite preparation. In fact, the passivation process can be used in order to obtain a good dispersion of metal nanoparticles in the polymeric matrix and to prevent the formation of the aggregate systems in the nanocomposites, thus improving mechanical properties and allowing them to tune the optical ones $[16,17]$.

Generally, two different types of nanocomposite morphologies can be achieved: (i) uniform distribution of non aggregate nanoparticles, (ii) nanoparticles distributed in the form of large aggregates [17].

It is also well-known that a good affinity between organic shell on nanoparticle surfaces and the polymer matrix leads to the first type of morphology, while non-uniform distribution is obtained when the organic shell is characterized by repulsive electrostatic interaction with the polymer matrix [17].

Aggregated and non-aggregated nanoparticles are characterized by different optical properties. In the case of contact-free metal nanoparticles system embedded in a dielectric medium such as the polymeric matrix, the material is characterized by a single surface plasmon absorption in the UV-Vis region of the spectrum, which originates from the resonant collective oscillations of the conduction electrons along the transversal direction of the electromagnetic field [18-20].

In an aggregates system, the dipole-dipole interactions between nanoparticles result in surface plasmon band broadening of extinctions spectrum caused by the contribution of both transversal and longitudinal electronic oscillation, i.e., along the direction of the electromagnetic field propagation. In the case of larger aggregates, the effect of the near charges is even more intensive and the broadening depends on the aggregate size and shape.

In this paper, an extensive study, concerning the interaction among in-situ synthesized thiol-capped silver nanoparticles embedded in a polymeric matrix obtained by thermal decomposition of the silver(I)-thiolates different types $\left(\mathrm{AgS}\left(\mathrm{CH}_{2}\right)_{n} \mathrm{X}\right.$, where $n$ is the number of carbon atoms that form the chain length and $X$ symbolizes the terminal group of the chain), is reported [21-23]. The influence of thiol structure on the interaction between adjacent nanoparticles was studied and characterization of samples obtained from different precursors by X-Ray diffraction (XRD) transmission electron microscopy (TEM), differential scanning calorimetric (DSC) and UV-Vis optical spectroscopy was reported.

\section{Results and Discussion}

\subsection{Structural and Morphological Characterizations}

The formation of silver nanoparticles inside the polymeric matrix was confirmed by XRD measurements. The recorded spectra of all samples were characterized by the typical XRD diffraction peaks assigned to the most abundant crystallographic planes of pure silver (JCPDS 87-0597). The Figure 2 shows, for the sake of simplicity, only two XRD spectra: the spectrum of the nanocomposite obtained by thermal decomposition of silver n-dodecyl-mercaptide (see Figure 2(a)) and of the silver cyclohexyl-mercaptide (see Figure 2(b)). All the XRD spectra of the samples obtained by thermal decomposition of linear precursor had a similar behavior to the first nanocomposite as shown in Figure 2(a). 
Figure 2. (a) X-Ray Diffraction (XRD) spectrum of nanocomposite obtained by thermal decomposition of silver n-dodecyl-mercaptide and (b) spectrum of nanocomposite obtained by thermal decomposition of silver cyclohexyl-mercaptide. The insets show the curve fitting of [111] diffraction peaks used to calculate the peak width at half-height for nanoparticles' size estimation from Scherrer's equation.
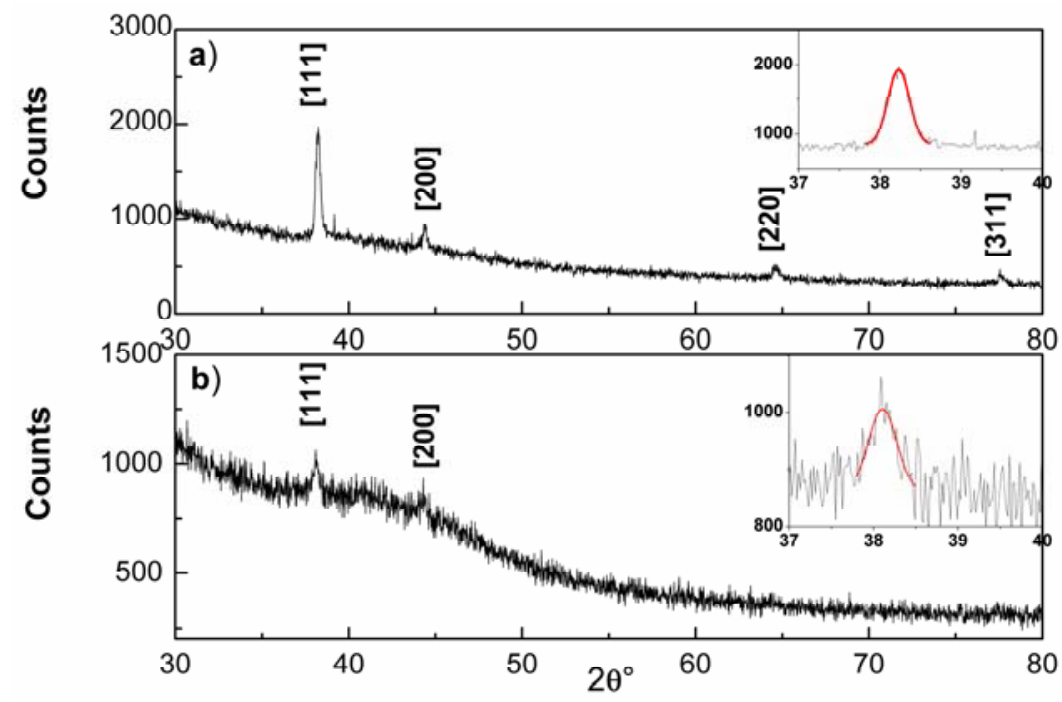

The significant diffraction peak broadening and the corresponding low intensity of the signal were due to the small size of the nanoparticles. In particular, the peak broadening, being due to the nanoparticles' size, is inversely proportional to nanoparticle size. According to Scherrer's formula $d=k \lambda /(\beta \cos \theta)$ where $\mathrm{k}=0.89$ (shape factor in the case spherical nanoparticles), $\lambda=1.54056 \AA$ (X-ray wavelength for $\mathrm{CuK} \alpha$ ) and $\beta$ is line broadening at half the maximum intensity (FWHM) in radians [21], the average dimension of the nanoparticles, evaluated from the [111] peak broadening was estimated as $d=6.5 \pm 0.2 \mathrm{~nm}$ for the samples obtained by thermal decomposition of silver n-dodecyl-mercaptide (see inset Figure 2(a)) and $d=3.5 \pm 0.8 \mathrm{~nm}$ in the case of thermal decomposition of silver cyclohexyl-mercaptide (see inset Figure 2(b)).

The XRD spectrum provided a direct confirmation that the precursor, i.e., silver(I) thiolate, completely decomposed the in-situ polymeric matrix, under the annealing process to form silver nanoparticles since its characteristic diffraction peak was absent.

Since XRD measurements may lead to an overestimation of the size of small nanoparticles, transmission electron microscopy (TEM) was employed to obtain a more accurate of the size estimate.

The nanoparticles' size and shape evaluation was obtained by processing TEM micrographs of different samples (Sigma Scan Pro 5 software). Figure 3 shows the size distribution histograms and nanocomposites TEM micrographs obtained by thermal decomposition of silver $n$-dodecyl-mercaptide and by silver cyclohexyl-mercaptide, respectively.

A typical TEM micrograph of the sample obtained by thermal decomposition of silver n-dodecyl-mercaptide shows the formation of spherical nanoparticles with average size of $d=4 \pm 0.4 \mathrm{~nm}$. Nanoparticles were organized in aggregate systems but not sintered together. In other words, the in situ method used to prepare the nanocomposites and the presence of thiol shell on the surface of silver nanoparticles avoids the coalescence phenomenon. The inset in Figure 3(c) shows a detail of 
nanoparticles' aggregate. The light grey areas inside the aggregate sketch the well separated nanoparticles represented by dark grey areas.

Figure 3. (a) and (b) Size distribution histogram of the nanocomposite obtained by thermal decomposition of silver n-dodecyl-mercaptide and of silver cyclohexyl-mercaptide, respectively. (c) and (d) The Transmission Electron Microscopy (TEM) micrograph of nanocomposites. The inset shows a detail of nanoparticles aggregate and marked by red lines the interparticles distance, $\boldsymbol{D}_{\boldsymbol{p} \boldsymbol{p}}$.
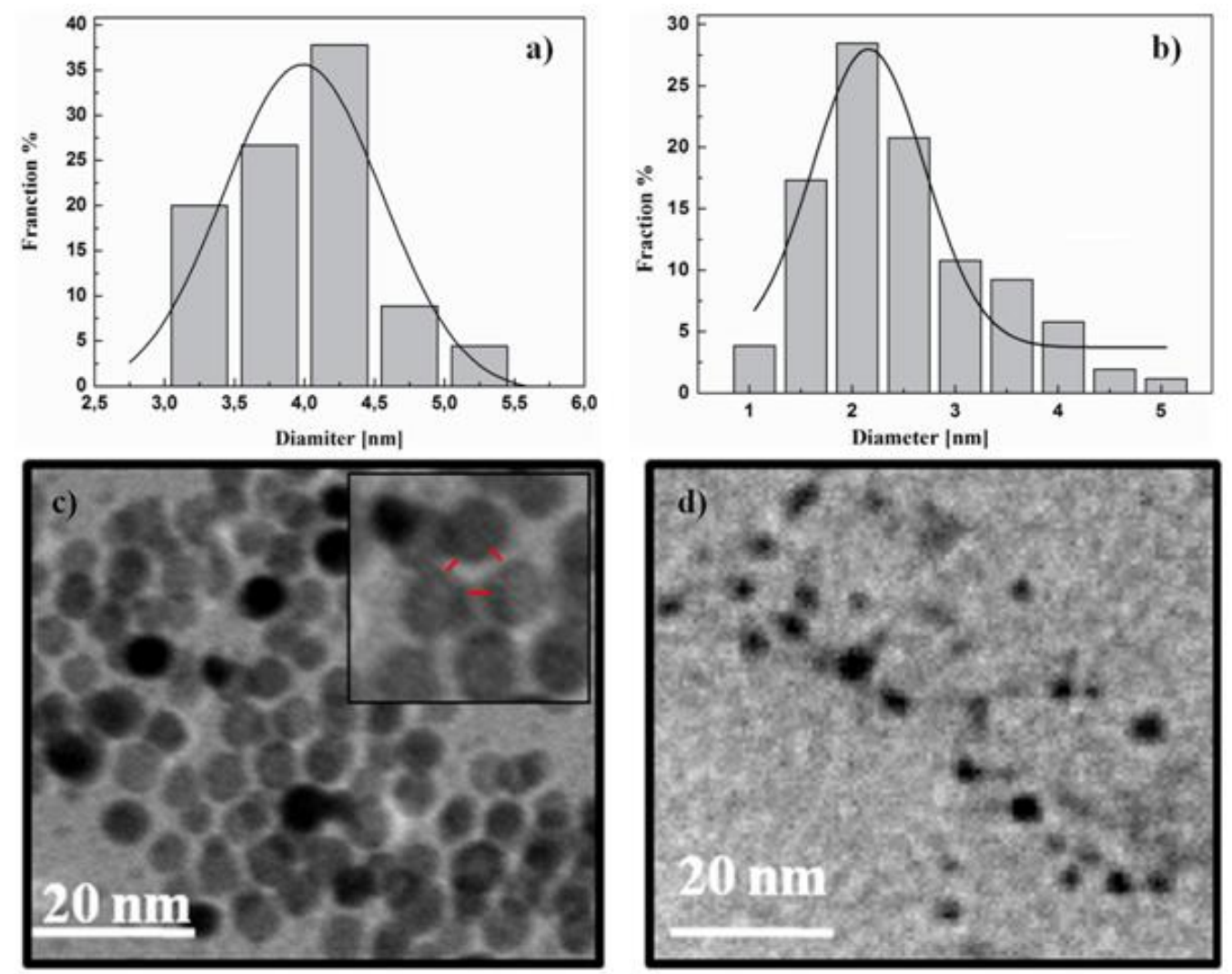

The average measured distance between adjacent ligand-capped nanoparticles was $\boldsymbol{D}_{\boldsymbol{p}}=1.5 \pm 0.3 \mathrm{~nm}$ (see red lines in inset) and it was shorter than $2 \boldsymbol{D}_{\boldsymbol{l}}=4 \mathrm{~nm}$ which was calculated assuming complete trans (zigzag) conformation [2]. Therefore, it is possible to deduce that the spacing into the aggregation system is controlled by interdigitation phenomena.

All the microstructures of the sample obtained by thermal decomposition of linear precursor had a similar morphological organization.

A fairly different morphological organization of silver nanoparticles was observed in the samples obtained by thermal decomposition of silver cyclohexyl-mercaptide (see Figure 3(d)).

TEM investigation evidenced a uniform dispersion of contact-free spherical nanoparticles with average size $d=2.1 \pm 0.29 \mathrm{~nm}$ (see Figure 3(b)). The significant difference with crystallite size value determined by TEM should be related to the negligible contribution that very small crystals gave to the $\mathrm{X}$-ray scattering.

Figure 3(d) shows the nanoparticles capped by thiol with cyclic structure. The encumbrance cyclic structure prevents interdigitation phenomenon and the formation of aggregate systems. 
These results confirmed the schematic representation of the proposed different interaction modes between silver nanoparticles capped by linear (left) as well as by cyclic structures (right) reported in Figure 1.

In addition, they showed that it was possible to control nanocomposites' morphology by using the different types of silver(I)-thiolates as precursor. Non uniform distribution characterized by the nanoparticle aggregates was obtained by using linear silver(I)-thiolates, uniform distribution was produced by cyclic silver(I)-thiolates.

Further structural analysis was performed by DSC analysis (see Figure 4). A first-order phase transition corresponding to the polystyrene glass transition temperature (Tg) at $c a .94{ }^{\circ} \mathrm{C}$ is clearly detected in the DSC data. However, an endothermic peak can be observed by increasing the temperature in the DSC thermograms of the samples obtained by thermal treatment of linear mercaptides (see Figure $4(\mathrm{a}-\mathrm{c})$ ). These peaks, centered at $c a .120{ }^{\circ} \mathrm{C}$, have been attributed to the collapse of $-\left(\mathrm{CH}_{2}\right)_{\mathrm{n}}$ - groups that form the organic capping layer [22-25]. The end of the interdigitation between thiols chains of neighboring nanoparticles takes place when the collapse occurs. In addition, the DSC measurement provided a confirmation for the presence of thiol-capped silver nanoparticles into the prepared samples.

Figure 4. DSC-thermograms of samples obtained by thermal decomposition of different mercaptides in amorphous polystyrene. (a) Silver n-dodecyl-mercaptide; (b) Silver n-hexadecyl-mercaptide; (c) Silver n-octadecyl-mercaptide; (d) Silver 11-mercapto-1undecacylmercaptide (e) Silver cyclohexyl-mercaptide.

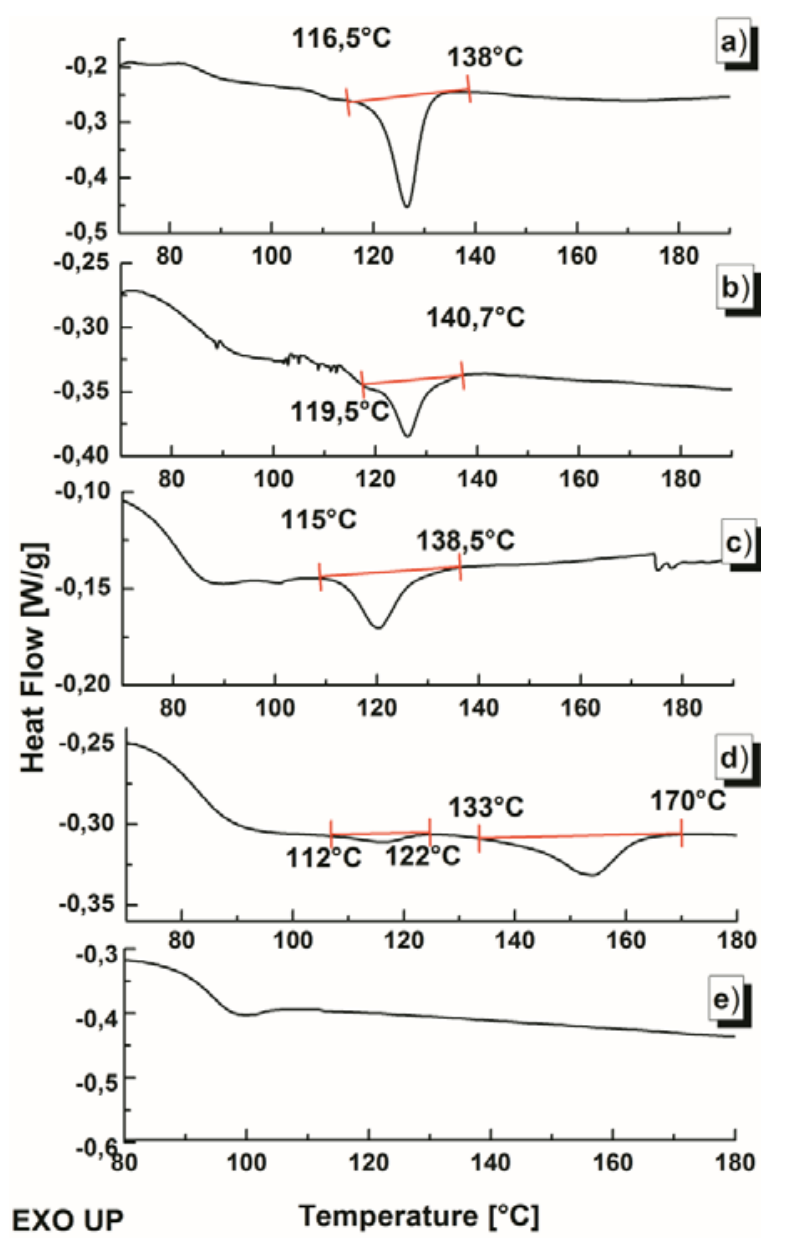


The DSC thermogram of the sample obtained by thermal decomposition of Ag-11-mercapto-1undecacylmercaptide showed (see Figure 4(d)) two different endothermic peaks. The peak at the lower temperature corresponded to the collapse of $-\left(\mathrm{CH}_{2}\right)_{\mathrm{n}}-$ groups present in the organic capping layer, whereas the second peak at the higher temperature can be attributed to the collapse of the interaction between thiol chains on neighbor nanoclusters caused by presence of $-\mathrm{OH}$ terminal groups.

Table 1 lists the temperature range of the endothermic peaks and the enthalpy values in the examined phase transitions.

Table 1. List of silver(I)-thiolates.

\begin{tabular}{cccc}
\hline Name & Molecular Formula & $\begin{array}{c}\text { Order-Disorder } \\
\text { Transition Temperature range }\left({ }^{\circ} \mathbf{C}\right)\end{array}$ & $\begin{array}{c}\Delta \mathbf{H} \\
(\mathbf{J} / \mathbf{g r})\end{array}$ \\
\hline Silver n-dodecyl-mercaptide & $\mathrm{CH}_{3}\left(\mathrm{CH}_{2}\right)_{11} \mathrm{SAg}$ & $116.5-138.0$ & 1.652 \\
Silver n-hexadecyl-mercaptide & $\mathrm{CH}_{3}\left(\mathrm{CH}_{2}\right)_{15} \mathrm{SAg}$ & $119.5-140.7$ & 2.962 \\
Silver n-octadecyl-mercaptide & $\mathrm{CH}_{3}\left(\mathrm{CH}_{2}\right)_{11} \mathrm{SAg}$ & $115.0-138.5$ & 2.865 \\
Silver 11-mercapto-1-undecacylmercaptide & $\mathrm{HO}\left(\mathrm{CH}_{2}\right)_{11} \mathrm{SAg}$ & $133.0-170.1$ & 1.895 \\
& & $(112.0-122.0)$ & $(0.300)$ \\
Silver cyclohexyl -mercaptide & $\mathrm{C}_{6} \mathrm{H}_{12} \mathrm{SAg}$ & - & - \\
\hline
\end{tabular}

The sample obtained by thermal decomposition of silver cyclohexyl-mercaptide (see Figure 4(e)) exhibited a quite different thermal behavior. The absence of endothermic peak after glass transition indicated that the cyclic thiol absorbed on metal nanoparticle surface did not allow for the interdigitation phenomena between neighboring nanoparticles.

\subsection{Optical Characterization}

Extinction spectra of linear and cyclic precursors displayed different features. Extinction spectra of the samples obtained by thermal decomposition of linear precursor were characterized by a broad surface plasmon absorption peak, whereas a narrow and symmetric peak appeared in the spectrum of the sample obtained by thermal decomposition of cyclic precursor.

Figure 5(a) displays the broad absorption surface plasmon resonance peak of nanocomposites obtained by thermal decomposition of linear silver n-dodecyl-mercaptide. The broadening of the peak is caused by the presence of aggregates nanoparticles, clearly seen in TEM images of the sample (see inset of Figure 3(a)).

As previously discussed, the dipole-dipole interactions between nanoparticles result in surface plasmon band broadening of extinctions spectrum caused by the contribution of both transversal and longitudinal electronic oscillation [18]. The contribution of the transversal and longitudinal electronic oscillations was determined by convolving the full extinction spectrum with two Gaussian functions (green curves in Figure 5(a)), centered at different wavelengths, the longer one corresponding to the longitudinal oscillations of the nanoparticles (i.e., $\lambda_{1}=575 \mathrm{~nm}$ ), the shorter to the transversal oscillations (i.e., $\lambda_{\mathrm{t}}=439 \mathrm{~nm}$ ). The red curve was obtained by adding the two contributions and smoothly interpolating the experimental data (dark line-square symbol). 
Figure 5. (a) and (b) Extinction spectra of the nanocomposite obtained by thermal decomposition of silver n-dodecyl-mercaptide and by silver cyclohexyl-mercaptide, respectively. Red curves represent the curve fitting to the experimental recorded spectra (black line-symbol) results. The fit was obtained with two Gaussian functions, green curves in (a) and with single Gaussian function in (b).
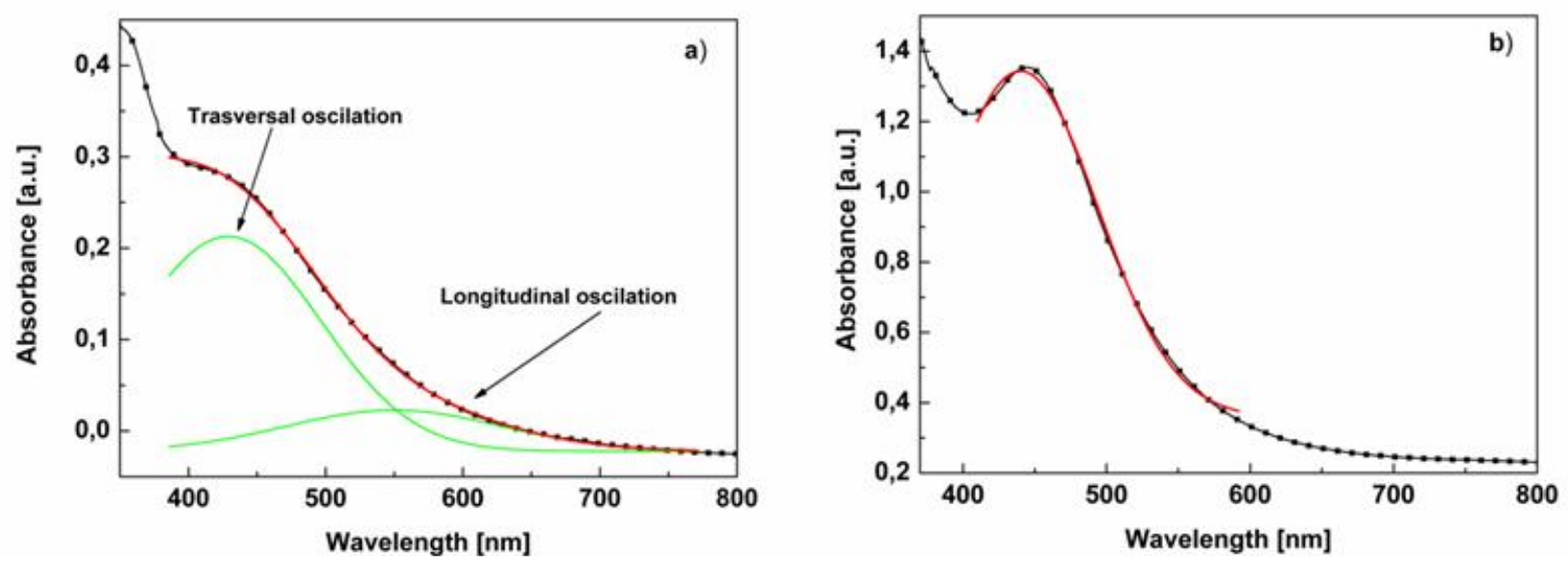

Compared to the case of the linear precursor, the extinction spectrum of the nanocomposite obtained by cyclic precursor (Figure 5(b)) shows a narrow peak at wavelength $\lambda_{t}=450 \mathrm{~nm}$. In this case the red fit curve was obtained by convolving the full extinction spectrum with single Gaussian function. This is a clear indication of the uniform dispersion of thiol-capped silver nanoparticles in polystyrene matrix.

\section{Experimental Section}

Thiol-capped silver nanoparticles in polymeric matrix were synthetized by in-situ thermal decomposition of different types of silver(I)-thiolates $\mathrm{AgS}\left(\mathrm{CH}_{2}\right)_{\mathrm{n}} \mathrm{X}$ [26-28]. We used linear thiolates with diverse terminal groups and non linear silver(I)-thiolates (see Figure 6).

Table 1 summarizes the compounds used as precursor of thiol-capped silver nanoparticles. Since these compounds are not commercially available, they were synthetized in the laboratory.

Basically, the procedure to obtained capped-thiol silver nanoparticles in polymeric matrix requires three steps: (i) synthesis of silver(I)-thiolates, (ii) preparation of silver(I)-thiolates/polymer blends, (iii) thermal annealing of the blends by using a special heating device.

The silver(I)-thiolates/polymer blends were obtained by drying, for 2 days at room temperature, a mixture of $\mathrm{AgS}\left(\mathrm{CH}_{2}\right)_{\mathrm{n}} \mathrm{X}$ and polystyrene (PS, Aldrich, $\mathrm{M}_{\mathrm{w}}=230,000 \mathrm{~g} \mathrm{~mol}^{-1}$ ) dissolved in chloroform. All samples were prepared using a 1:10 $\mathrm{AgS}\left(\mathrm{CH}_{2}\right)_{\mathrm{n}} \mathrm{X} / \mathrm{PS}$ weight ratio. Then, the blends were isothermally annealed at $200{ }^{\circ} \mathrm{C}$ for $3 \mathrm{~min}$ by using a specially designed laboratory hot-plate furnace, equipped with a thermocouple and connected to an automatic temperature controller, Aldrich DigiTrol II. The developed furnace is made of a large metallic mono-block which can be uniformly heated, limiting the temperature gradients (both longitudinal and transversal to the film surface) into the samples during the thermolysis process [16,26-28]. 
Figure 6. Schematic representation of different types of silver(I)-thiolates.
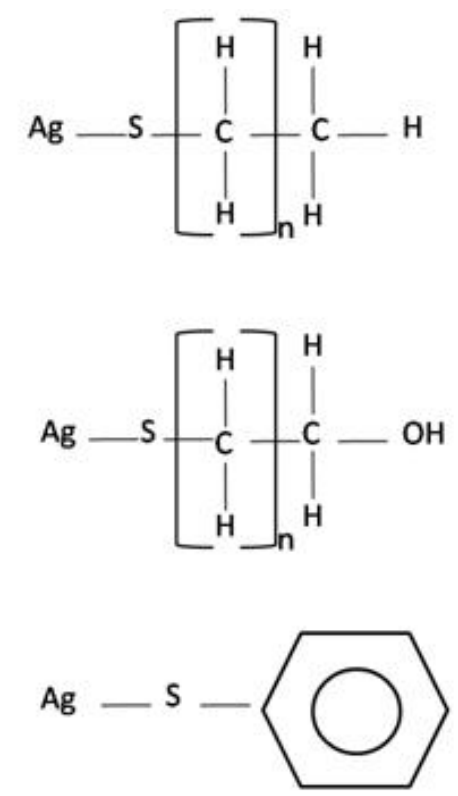

Among different optical polymers (i.e., transparent amorphous polymers with refractive index close to 1.5), we selected the amorphous polystyrene (PS) (Aldrich, $\mathrm{M}_{\mathrm{w}}=230,000 \mathrm{~g} \mathrm{~mol}^{-1}, \mathrm{~T}_{\mathrm{g}} \cong 94{ }^{\circ} \mathrm{C}$ ) for the nanocomposites development. In fact, PS is one of the most used optical plastic (transparent in the visible range, with 1.589 refractive index), due to its low cost and good solubility in a wide range of no polar solvents.

The reaction mechanism for the formation of thiol-capped silver nanoparticles follows three phases: (i) thermal decomposition of silver(I)-thiolates, (ii) nucleation and growth of nanoparticles, (iii) passivation by chemical absorption on the silver nanoparticles surface of dialkyl disulfides $\left(\mathrm{SR}_{\mathrm{m}}\right)$ by-product of reaction. The disulfide molecules chemically bind the silver nanoparticles surface by Ag-S covalent bonds lead to form an organic shell on surface silver nanoparticles.

The structure of the metallic phase product was investigated by X-Ray Diffraction (XRD) using a Rigaku DMAX-IIIC diffractometer with $\mathrm{CuK} \alpha$ radiation $(\lambda=0.154 \mathrm{~nm})$. The morphological characterization was performed by means of Transmission Electron Microscopy (TEM), using a Philips EM208S microscope, operating at an accelerating voltage of $100 \mathrm{kV}$ and equipped with a MegaView Camera for digital imaging. The specimens for TEM investigation were prepared by dissolving the nanocomposites in chloroform, the solutions so obtained being added drop wise onto TEM cooper mesh grids.

The crystalline packing of the organic shell on the surface nanoparticles in polymeric matrix was proved by the Differential Scanning Calorimetry (DSC, TA instrument 2920). Samples of few milligrams were sealed into hermetic aluminum pans and DSC tests were done under a purging atmosphere of nitrogen gas at rates of $10{ }^{\circ} \mathrm{C} / \mathrm{min}$.

Optical properties of the produced nanocomposites were examined by recording the absorption spectra using an UV-Visible spectrophotometer (Perkin-Elmer-Lambda 850).

The instrument allows the direct investigation of the as-prepared samples as film specimens. Double beam UV-Visible spectrophotometer allows the simultaneous recording of the extinction spectra of the sample and reference. The reference spectrum is subtracted from that of the sample. 


\section{Conclusions}

In this paper, the synthesis method of thiol-capped silver nanoparticles was based on the thermal decomposition of different silver(I)-thiolate precursors into a transparent polymeric matrix. Two different types of nanoparticle morphology were observed due to the chemical structure of the coating-precursors, that is, the possibility to crystallize by interdigitation. In particular, nanoparticles' aggregate systems were obtained using linear precursors. Due to the interdigitation phenomenon, the uniform distribution of contact-free nanoparticles was achieved with cyclic precursors.

\section{References}

1. Schmid, G. Nanoparticles; WILEY-VCH: Weinheim, Germany, 2004.

2. Feldheim, D.L.; Foss, C.A., Jr. Metal Nanoparticles: Synthesis, Characterization, and Applications; Marcel Dekker: New York, NY, USA, 2001.

3. Rao, C.N.R.; Müller, A.; Cheetam, A.K. The Chemistry of Nanaomaterials; WILEY-VCH: Weinheim, Germany, 2004.

4. Koch, C.C. Nanostructured Materials, Processing, Properties and Potential; William Andrew Publishing: Norwich, UK, 2002.

5. Ruckenstein, E.; Li, Z.F. Surface Modification and Functionalization through the Self-Assembled Monolayer and Graft Polymerization. Adv. Colloid. Interface Sci. 2005, 113, 43-63.

6. Kickelbick, G.; Schubert, U. Organic Functionalization of Metal Oxide Nanoparticles. In Functionalization and Surface Treatment of Nanoparticles; Baraton, M.-I., Ed.; American Scientific Publishers: Stevenson Ranch, CA, USA, 2003; p. 91.

7. Pukanszky, B.; Fekete, E. Adhesion and Surface Modification, Adv. Polym. Sci. 1999, 139, 109-153.

8. Grancharov, S.G.; Zeng, H.; Sun, S.; Wang, S.X.; O’Brien, S.; Murray, C.B.; Kirtley, J.R.; Held, G.A. Bio-Functionalization of Monodisperse Magnetic Nanoparticles and Their Use as Biomolecular Labels in a Magnetic Tunnel Junction Based Sensor. J. Phys. Chem. B Condens. Matter. Mater. Surf. Interfaces. Biophys. 2005, 109, 13030-13035.

9. Schimd, G. Large Clusters and Colloids. Metals in the Embryonic State. Chem. Rev. 1992, 92, 1709-1727.

10. Giersig, M.; Mulvaney, P. Preparation of Ordered Colloid Monolayers by Electrophoretic Deposition. Langumir 1993, 9, 3408-3413.

11. Brust, M.; Walker, M.; Bethell, D.; Schiffrin, D.J.; Whyman, R. Synthesis of Thiol Derivatised Gold Nanoparticles in a Two Phase Liquid/Liquid System. J. Chem. Soc. Chem. Commun. 1994, 801-802.

12. Brust, M.; Kiely, C.J. Monolayer Protected Clusters of Gold and Silver. In Colloids and Colloid Interfaces; Caruso, F., Ed.; Wiley-VCH: Weinheim, Germany, 2004.

13. Gooding, J.J.; Mearns, F.; Yang, W.; Liu, J. Self-Assembled Monolayers into the 21st Century: Recent Advances and Applications. Electroanalysis 2003, 15, 81-96.

14. Lide, D.R. Handbook of Chemistry and Physic, 82nd ed.; CRC Press: Boca Raton, FL, USA, 2001. 
15. Doty, R.C.; Tshikhudo, T.R.; Brust, M.; Fernig, D.G. Extremely Stable Water-Soluble Ag Nanoparticles. Chem. Mater. 2005, 17, 4630-4635.

16. Nicolais, L.; Carotenuto, G. Metal-Polymer Nanocomposite; John Wiley \& Sons Inc.: Hoboken, NJ, USA, 2005.

17. Rozenberga, B.A.; Tenneb, R. Polymer-Assisted Fabrication of Nanoparticles and Nanocomposites. Prog. Polym. Sci. 2008, 33, 40-112.

18. Kreibig, U.; Vollmer, M. Optical Properties of Metal Clusters; Springer-Verlag: Berlin, Germany, 1995.

19. Haberland, H. Clusters of Atoms and Molecules; Springer: Berlin, Germany, 1994.

20. Bohren, C.F.; Huffman, D.R. Absorption and Scattering of Light by Small Particles; Wiley: New York, NY, USA, 1983.

21. Patterson, A.L. The Scherrer Formula for X-Ray Particle Size Determination. Phys. Rev. 1939, 56, 978-982.

22. Benseba, F.; Zhou, Y.; Brolo, A.G.; Irish, D.E.; Deslandes, Y.; Kruus, E.; Ellis, T.H. Raman Characterization of Metal-Alkanethiolates. Spectrochim. Acta Part A 1999, 55, 1229-1236.

23. Ang, T.P.; Chin, W.S. Dodecanethiol-Protected Copper/Silver Bimetallic Nanoclusters and Their Surface Properties. J. Phys. Chem. B 2005, 109, 22228-22236.

24. Sandhyarani, N.; Predeep, T. Crystalline Solids of Alloy Clusters. Chem. Mater. 2000, 12, 1755-1761.

25. Han, S.W.; Kim, Y.; Kim, K. Dodecanethiol-Derivatized Au/Ag Bimetallic Nanoparticles: TEM, UV/VIS, XPS, and FTIR Analysis. J. Colloid Interface Sci. 1998, 208, 272-278.

26. Carotenuto, G.; Martorana, B.; Perlo, P.; Nicolais, L. A Universal Method for the Synthesis of Metal and Metal Sulfide Clusters Embedded in Polymer Matrices. J. Mater. Chem. 2003, 13, 2927-2930.

27. Nicolais, F.; Carotenuto, G. Synthesis of Polymer-Embedded Metal, Semimetal, or Sulfide Clusters by Thermolysis of Mercaptide Molecules Dissolved in Polymers. Recent Patents Mater. Sci. 2008, 1, 1-11.

28. Carotenuto, G.; Nicolais, L.; Perlo, P. Synthesis of Polymer-Embedded Noble Metal Clusters by Thermolysis of Mercaptides Dissolved in Polymers. Polym. Eng. Sci. 2006, 46, 1016-1020.

(C) 2011 by the authors; licensee MDPI, Basel, Switzerland. This article is an open access article distributed under the terms and conditions of the Creative Commons Attribution license (http://creativecommons.org/licenses/by/3.0/). 